\title{
Exercise intervention alters HDL subclass distribution and function in obese women
}

\author{
Nicholas J Woudberg ${ }^{1 *}$, Amy E Mendham ${ }^{2,4}$, Arieh A Katz ${ }^{3}$, Julia H Goedecke ${ }^{2,4}$ and Sandrine Lecour ${ }^{1}$
}

\begin{abstract}
Background: Obesity is associated with a change in high-density lipoprotein (HDL) function and subclass. Exercise training reduces cardiovascular risk in obese patients. We aimed to explore the effect of an exercise training stimulus on HDL functionality and subclass in obese women.

Methods: Thirty-two obese black South African women were randomly assigned to exercise (combined aerobic and resistance exercise) or control (no exercise) conditions for 12-weeks. Pre- and post-testing included venous blood sampling for analysis of lipid profile and HDL functionality, by measuring cellular cholesterol efflux capacity, reduction in endothelial vascular cell adhesion molecule (VCAM) expression (anti-inflammatory function), paraoxonase (PON) (antioxidative function) and platelet activating factor acetylhydrolase (PAF-AH) activities (antithrombotic function). PON-1 and PAF-AH expression were determined in serum and in isolated HDL using Western blotting. Levels of large, intermediate and small HDL subclasses were measured using the Lipoprint ${ }^{\circledast}$ system.

Results: Exercise training resulted in a decrease in body mass index $(-1.0 \pm 0.5 \% \mathrm{vs}+1.2 \pm 0.6 \%, p=0.010)$, PON activity $(-8.7 \pm 2.4 \%$ vs $+1.1 \pm 3.0 \%, p=0.021)$, PAF-AH serum expression $(-22.1 \pm 8.0 \%$ vs $+16.9 \pm 9.8, p=0.002)$, and the distribution of small HDL subclasses $(-10.1 \pm 5.4 \% \mathrm{vs}+15.7 \pm 6.6 \%, p=0.004)$ compared to controls. Exercise did not alter HDL cellular cholesterol efflux capacity and anti-inflammatory function.

Conclusions: These results demonstrate the potential for exercise training to modify HDL subclass distribution and $\mathrm{HDL}$ function in obese women.

Trial registration: Clinical trials number: PACTR201711002789113.

Keywords: Obesity, Exercise intervention, HDL structure, Cholesterol efflux, HDL subclass, Antioxidative, Antiinflammatory
\end{abstract}

\section{Background}

Approximately $23 \%$ of the worldwide burden of ischemic heart disease can be attributed to obesity, with the prevalence of obesity doubling since 1980 [1]. Exercise is a popular intervention designed to treat obesity and prevent the onset of associated non-communicable diseases [2]. Indeed, exercise training interventions (aerobic and/or resistance) reduce cardiovascular risk factors such as body fat mass, blood pressure, total cholesterol, low-density lipoprotein cholesterol (LDL-C) and raise high-density lipoprotein cholesterol (HDL-C) [2-7]. As little as $30 \mathrm{~min}$ of exercise per day can increase the concentration of

\footnotetext{
* Correspondence: nicholaswoudberg@gmail.com

${ }^{1}$ Hatter Institute for Cardiovascular Research in Africa, Department of Medicine, Faculty of Health Sciences, University of Cape Town, Cape Town, South Africa

Full list of author information is available at the end of the article
}

HDL-C in diabetic patients [8]. Although HDL-C concentrations are inversely associated with risk for cardiovascular disease [9], the recent outcomes of clinical trials aimed at reducing the risk of cardiovascular disease (CVD) by increasing HDL-C levels have been unsuccessful (reviewed by [10]). Accordingly, there has been a significant shift in focus from studying the quantity of HDL to studying the quality [11-15]. HDL quality refers to specific HDL functions and the distribution of HDL subclasses.

Although the principal function of HDL is reverse cholesterol efflux/transport (RCT), HDL displays additional physiological functions including antioxidative, anti-inflammatory and anti-thrombotic activities. These are mediated, at least in part, by HDL-associated enzymes such as paraoxonase (PON) (antioxidative) and platelet activating factor acetylhydrolase (PAF-AH)

(C) The Author(s). 2018 Open Access This article is distributed under the terms of the Creative Commons Attribution 4.0 International License (http://creativecommons.org/licenses/by/4.0/), which permits unrestricted use, distribution, and 
(anti-thrombotic) [16, 17]. HDL can also be subdivided into several subclasses which display distinct functionalities [18].

The African black population has low prevalence of coronary artery and ischemic heart disease, which was originally attributed to higher serum HDL-C [19]. Interestingly, studies highlight that HDL-C is the same or even lower in black compared to white populations [19-22]. A more recent study has further shown that HDL functionality in African black women differed from their white counterparts, with black women displaying a higher level of HDL antioxidative function [22]. These data support the notion that HDL quality, rather than quantity may account for the difference in prevalence of CVD between the white and black populations. Furthermore, exercise training may influence HDL function [23]; however, the impact of exercise training on HDL quality in a black population is still unclear [23]. The aim of the study was to examine the effects of exercise training on HDL functionality and subclass, in obese black South African women.

\section{Methods}

Full methodology regarding recruitment and testing is detailed by [24].

\section{Participants}

Forty-five women were recruited during 2015 and 2016 from the Western Cape, South Africa. Inclusion criteria were: $20-35$ years in age, obese (BMI $30-40 \mathrm{~kg} / \mathrm{m}^{2}$ ), weight stable for 6 months, black South African (both biological parents isiXhosa), sedentary (not participating in exercise training $(<1$ session of $<20$ min per week) for a minimum of 12 months, on injectable contraceptive (depot medroxyprogesterone acetate, $400 \mathrm{mg}$ ) for a minimum of 2 months, no known illness or chronic disease, not taking any medications, and had no surgical procedures within the last 6 months. This study was approved by the Human Research Ethics Committee at the University of Cape Town (HREC REF: 054/2015), complies with Declaration of Helsinki principles and participants provided written consent prior to testing. Clinical trials number: PACTR201711002789113.

\section{Study design}

Participants were block randomized into either control $(n=22)$ or exercise $(n=23)$ conditions (Additional file 1: Figure S1). Thirty-five women in control $(n=15)$ and exercise $(n=20)$ groups completed the study. The exercise intervention consisted of 12 weeks of supervised aerobic and resistance exercise training of moderatevigorous intensity for 40 to $60 \mathrm{~min}, 4$ days per week by a trained exercise physiologist. Exercises included cardiovascular exercises in the form of aerobic dance, running, skipping, and stepping that were performed at a moderate-vigorous intensity $(75-80 \%$ peak heart rate, $\left.\mathrm{HR}_{\text {peak }}\right)$. Resistance exercises included the participants using their own body weight and progressed to the use of equipment (eg bands and free weights). These exercises included squats, lunges, bicep curls, push-ups and shoulder press with a prescribed intensity of $60 \%$ to $70 \% \mathrm{HR}_{\text {peak }}$. Participants wore a heart rate monitor (Polar A300, Kempele, Finland) during all training sessions to ensure the prescribed intensity was maintained throughout the 12 -week period. The control participants were instructed to continue their normal physical activity and dietary patterns. Participants attended two pre- and two post-intervention testing sessions. The first session comprised of anthropometry and a graded exercise test for the assessment of peak oxygen consumption $\left(\mathrm{VO}_{2 \text { peak }}\right)$ and peak heart rate $\left(\mathrm{HR}_{\text {peak }}\right)$. After a minimum of 48 -h recovery from the previous testing session, participants returned for fasting (10-12 h) venous blood collection for analysis of total cholesterol (total-C), low-density lipoprotein cholesterol (LDL-C), HDL-C concentration, HDL functionality and subclass distribution.

\section{Nutritional and physical activity standardization}

The exercise and control groups were instructed to maintain their usual dietary intake. The control group was also instructed to continue their habitual physical activity and to refrain from initiating any exercise program. Prior to the start of all testing sessions, participants refrained from any physical activity for a minimum of $48 \mathrm{~h}$, and from consumption of alcohol and caffeine for $24 \mathrm{~h}$.

\section{Anthropometry and graded exercise test}

Anthropometric measures included stature, body mass, waist (at level of umbilicus) and hip girths (greatest protrusion of buttocks) using standard techniques [25]. These measures were used to calculate body mass index (BMI) and the waist-to-hip ratio (WHR). $\mathrm{VO}_{2 \text { peak }}$ and $\mathrm{HR}_{\text {peak }}$ (Polar A300, Kempele, Finland) were measured using a treadmill-based (C, Quasar LE 500 CE, HP Cosmos, Nussdorf-Traunstein, Germany) graded exercise test. This walking cardiorespiratory fitness test was designed for participants whom were sedentary and unfamiliar with gym-based equipment. Pulmonary gas exchange was measured by determining $\mathrm{O}_{2}$ and $\mathrm{CO}_{2}$ concentrations and ventilation to calculate $\mathrm{VO}_{2}$ consumption using a metabolic gas analysis system (CPET, Cosmed, Rome Italy). Prior to each test, the gas meter was calibrated with a 3-1 syringe (Vacumed, Ventura, CA), and analyzers calibrated using standard gas mixtures of oxygen $\left(26 \% \mathrm{O}_{2}\right.$ with the balance nitrogen) and carbon dioxide $\left(4 \% \mathrm{CO}_{2}, 16 \% \mathrm{O}_{2}\right.$ and the 
balance nitrogen; BOC Special Gas, Afrox Cape Town, South Africa).

\section{Venous blood collection and lipid profile}

Fasting (10-12 h) venous blood samples were collected in serum separating tubes (SST) and clotted for 15-30 min at room temperature. Samples were centrifuged at $3000 \mathrm{rpm}$ for $10 \mathrm{~min}$ at $4{ }^{\circ} \mathrm{C}$. Serum was immediately stored at $-80{ }^{\circ} \mathrm{C}$ until further analyses. Serum lipid profile (HDL-C, LDL-C and Total-C) was determined using a colorimetric assay (Randox (Pty) Ltd., Gauteng, South Africa).

\section{HDL isolation}

HDL was isolated from aliquots of serum using ultracentrifugation, as previously described [26, 27]. Purity was confirmed using $12.5 \%$ reducing SDS-polyacrylamide gel electrophoresis (PAGE) stained with Coomassie Blue. The protein concentration of HDL was determined by the modified Lowry method [28] All samples were analysed in duplicate.

\section{Quantification of HDL anti-inflammatory function}

HDL anti-inflammatory function was measured using a cell culture model as previously described [27]. Briefly, human umbilical vein endothelial cells (HUVEC) were serum deprived prior to treatment with $10 \mu \mathrm{g} / \mathrm{ml}$ of isolated HDL for $30 \mathrm{~min}$. Eight participants per group were randomly selected (Additional file 1: Figure S1). Cells were then stimulated with $20 \mathrm{ng} / \mathrm{ml}$ murine tumour necrosis factor alpha (TNF- $\alpha$ ) (PeproTech, 315-01A) for 5 h. Following RNA isolation and cDNA synthesis, cDNA was amplified for 25 cycles using the RT2 SYBR Green qPCR kit (Qiagen, 330,500) in the RotorGene6000 (Corbit Lifesciences) to quantify expression levels of VCAM and GAPDH. Data is presented as relative reduction in VCAM expression compared to an untreated control.

\section{Quantification of HDL cellular cholesterol efflux capacity}

HDL induced cholesterol efflux was quantified using a modified method [29]. Briefly, RAW264.7 cells, generously donated by Prof Gil Dealtry (Nelson Mandela Metropolitan University), were proliferated in RPMI-1640 media (Sigma, R8758) supplemented with 10\% foetal calf serum and penicillin/streptomycin prior to seeding $(100,000$ cells/well) in 24-well culture plates for $16 \mathrm{~h}$. Labelling medium was prepared by adding $4 \mu \mathrm{Ci} / \mathrm{ml}$ of $\left[{ }^{3} \mathrm{H}\right]$ cholesterol (Perkin Elmer, NET139001MC) to RPMI-1640 medium containing $2 \mu \mathrm{g} / \mathrm{ml}$ of acyl-CoA cholesterol acyltransferase (ACAT) inhibitor (Sandoz, Sigma, S9318) and supplemented with $5 \%$ foetal calf serum. Cells were then incubated in labelling medium for $24 \mathrm{~h}$. Cells were washed with minimum essential eagle medium (MEM) in HEPES buffer prior to addition of $25 \mu \mathrm{g} / \mathrm{ml}$ of isolated HDL in MEM-HEPES for $4 \mathrm{~h}$. Cell culture media was extracted and added to Ultima Gold scintillant (Perkin Elmer, $6,013,327)$. Counts per minute (CPM) were enumerated using TriCarb ${ }^{\circ}$ Liquid Scintillation Analyzer and QuantaSmartTM software with 2 Sigma terminator 0.5 and $30 \mathrm{~min}$ count time. Cellular cholesterol efflux capacity was calculated as label present in the cell media relative to the untreated control.

\section{Paraoxonase (PON) activity}

Serum paraoxonase activity was measured as previously described [27]. Serum samples were diluted 1:10 in phosphate buffer containing $2 \mathrm{mM} \mathrm{CaCl} 2(\mathrm{pH} 8)$. Diluted serum was added to 96-well plates in triplicate and paraoxon-ethyl substrate (Sigma, D9286) was added. Absorbance at $\mathrm{A}_{405}$ was measured at $30 \mathrm{~s}$ intervals over $20 \mathrm{~min}$. One Unit of activity is defined as $1 \mathrm{nmol}$ of substrate hydrolysed per min.

\section{Platelet activating factor Acetylhydrolase (PAF-AH) activity}

PAF-AH activity was measured in participant sera as previously described [27], using the PAF Acetylhydrolase Assay Kit (Cayman Chemical, 760,901). Briefly, serum was added to an equal volume of 5, 5'-dithio-bis-(2-nitrobenzoic acid) (DTNB; Ellman's Reagent) and assay buffer in triplicate into clear 96-well plates. All wells were incubated with 2-thio PAF substrate and absorbance at $\mathrm{A}_{412}$ measured at $1 \mathrm{~min}$ time intervals for $20 \mathrm{~min}$. One Unit of activity is defined as $1 \mu \mathrm{mol}$ of substrate hydrolysed per min.

\section{PON-1 and PAF-AH expression}

Isolated HDL and serum samples from each of the participants were electrophoresed on reducing 12.5\% SDSpolyacrylamide (SDS-PAGE) gels with $1.5 \mu \mathrm{g}$ of HDL protein or $8 \mu \mathrm{g}$ of serum loaded per well. Samples were run over three separate gels with control samples repeated in each gel. Blots were transferred onto nitrocellulose membranes (Bio-Rad, 162-0113). Ponceau S staining was scanned and used to validate equal loading of wells. Blots were blocked in 5\% low fat milk powder in $0.05 \%$ Tween in Tris-buffered Saline (TTBS, pH 7.5) and incubated overnight in primary mouse anti-PON-1 antibody (1:200) [26] and rabbit anti-PAF-AH (1:400) (Cayman Chemical, 160,603). Blots were then washed in TTBS and incubated in goat anti-mouse-HRP conjugated secondary antibody (1:5000) (Bio-Rad, 170 6516) and goat anti-rabbit-HRP conjugated secondary antibody (1:2500) (Santa Cruz Biotechnology, sc-2313), respectively for $1 \mathrm{~h}$ at room temperature. Blots were thoroughly washed in TTBS prior to incubation in Amersham TM ECL ${ }^{\mathrm{TM}}$ Western blotting detection reagent (GE Healthcare, RPN2106). Blots were captured 
in the GeneGnome gel imager. Densitometry of PON-1 and PAF-AH blots was quantified using Quantity one software. PON-1 and PAF-AH relative expression data were corrected for control samples, repeated in each gel.

\section{Quantification of HDL subclass distribution}

Serum HDL subclass was determined using the Lipoprint $^{\circ}$ HDL system (Quantimetrix, Redondo Beach, CA) as previously described [27]. Briefly, serum $(25 \mu \mathrm{l})$ was mixed with Lipoprint loading gel, containing Sudan black dye which binds proportionally to the cholesterol present in the sample. The mix was placed onto the upper part of the high resolution $3 \%$ polyacrylamide gel. Photopolymerisation was carried out for $30 \mathrm{~min}$ at room temperature and electrophoresis was performed for $50-60 \mathrm{~min}$ at $3 \mathrm{~mA}$ per gel tube. After a rest period of $30 \mathrm{~min}$, gel tubes were scanned and analysed using the Lipoware software. For HDL subclass, the very low-density lipoprotein (VLDL) and LDL remained at the origin [Retention Factor $(\mathrm{Rf})=0.0$ ] and is shown as a grey peak to the left of the large HDL subclass distribution profile while albumin migrated as the leading front $(\mathrm{Rf}=1.0)$. Between these, $10 \mathrm{HDL}$ bands could be detected. HDL-1, HDL-2 and HDL-3 were defined as large HDL; HDL-4, HDL-5, HDL-6 and HDL-7 were defined as intermediate HDL and HDL-8, HDL-9 and HDL-10 were defined as small HDL. Each subclass was quantified and expressed as a percentage of total HDL.

\section{Statistical analysis}

Results are presented as mean or as percentage changes relative to baseline \pm standard error of mean (SEM). Non-normally distributed data were log transformed prior to statistical analysis and included LDL-C, serum PON-1, serum and HDL PAF-AH expression. Non-normally distributed data are presented as medians \pm interquartile range (IQR). Sample size determination was based on previous studies regarding exercise training interventions in obese individuals $[10,30]$, using a significance level of $p$ $<0.05$ and power of $80 \%$, as described in detail previously [24]. Two-way repeated measures analysis of variance was used to compare changes in anthropometry, $\mathrm{VO}_{2 \text { peak }}$, lipids, cholesterol efflux capacity, anti-inflammatory function, paraoxonase activity, PAF-AH activity and HDL subclass distribution between groups over the 12-week period, followed by Fischer post-hoc testing. Pearson correlation coefficients for the associations between anthropometry, $\mathrm{VO}_{2 \text { peak }}$, HDL-C, HDL function and subclass were determined at baseline and changes in the combined sample. Where appropriate, statistical analysis were adjusted for patient BMI. $p<0.05$ was deemed statistically significant and statistical tests were performed using Statistica (Version 13.2, Dell Inc., 2016).

\section{Results \\ Changes in anthropometry, cardiorespiratory fitness and lipids}

Adherence to the exercise training, expressed as the percentage attendance of total number of sessions, was $80.3 \pm 3.0 \%$ (range: 60.4-100\%). Exercise training resulted in a significant increase in cardiorespiratory fitness $\left(\mathrm{VO}_{2 \text { peak }}\right)$ compared to control $(p=0.003$ for interaction, Table 1). BMI, body weight, waist and hip circumference and waist/hip ratio (WHR) decreased in response to the 12-week intervention in the exercise group compared to the controls $(p<0.05$ for interaction). Total-C, HDL-C and LDL-C concentrations did not vary between groups in response to the intervention $(p=0.141, p=0.238$ and $p=0.202$ for interaction,

Table 1 Changes in anthropometry, cardiorespiratory fitness and lipid profile in response to the 12-week intervention

\begin{tabular}{|c|c|c|c|c|c|c|c|}
\hline & Control $(n=$ & & Exercise $(n=$ & & $p$ value & & \\
\hline & Pre-testing & Post-testing & Pre-testing & Post-testing & Group & Time & Interaction \\
\hline Age (yrs) & $24.5 \pm 0.9$ & & $22.8 \pm 0.7$ & & 0.157 & - & - \\
\hline BMI $\left(\mathrm{kg} / \mathrm{m}^{2}\right)$ & $33.3 \pm 0.8$ & $33.7 \pm 0.8$ & $34.4 \pm 0.6$ & $34.0 \pm 0.7$ & 0.493 & 0.678 & 0.010 \\
\hline Body weight (kg) & $88.0 \pm 3.2$ & $89.1 \pm 3.2^{*}$ & $84.1 \pm 1.9$ & $83.3 \pm 2.2$ & 0.187 & 0.681 & 0.007 \\
\hline Waist circumference $(\mathrm{cm})$ & $103 \pm 2$ & $106 \pm 2^{*}$ & $104 \pm 2$ & $100 \pm 2^{* *}$ & 0.406 & 0.548 & 0.001 \\
\hline Hip circumference $(\mathrm{cm})$ & $118 \pm 2$ & $119 \pm 2$ & $114 \pm 1$ & $113 \pm 1^{*}$ & 0.045 & 0.359 & 0.022 \\
\hline WHR & $0.88 \pm 0.01$ & $0.89 \pm 0.02$ & $0.91 \pm 0.01$ & $0.89 \pm 0.01$ & 0.436 & 0.892 & 0.044 \\
\hline Total-C (mmol/L) & $3.91 \pm 0.35$ & $3.65 \pm 0.35$ & $3.98 \pm 0.18$ & $4.27 \pm 0.21$ & 0.299 & 0.917 & 0.141 \\
\hline $\mathrm{LDL}-\mathrm{C}(\mathrm{mmol} / \mathrm{L})$ & $1.94 \pm 0.29$ & $1.57 \pm 1.43$ & $2.80 \pm 0.99$ & $2.69 \pm 1.34$ & 0.169 & $<0.001$ & 0.202 \\
\hline $\mathrm{HDL}-\mathrm{C}(\mathrm{mmol} / \mathrm{L})$ & $0.97 \pm 0.06$ & $0.97 \pm 0.06$ & $1.00 \pm 0.05$ & $1.05 \pm 0.04$ & 0.343 & 0.511 & 0.568 \\
\hline $\mathrm{VO}_{\text {2peak }}(\mathrm{ml} / \mathrm{kg} / \mathrm{min})$ & $23.7 \pm 0.8$ & $22.7 \pm 0.9$ & $25.2 \pm 0.7$ & $27.7 \pm 0.7^{*}$ & 0.002 & 0.202 & 0.003 \\
\hline
\end{tabular}

Results represent means \pm SEM and as medians \pm IQR for LDL-C. Unadjusted $p$ values testing for significance of the grouping variable (Control vs Exercise), time (intervention duration) and the interaction (Group*Time). For Fischer post-hoc testing following interaction effect: * $p<0.05 * *<<0.005$ pre vs post-testing. BMI Body mass index, WHR Waist/hip ratio, Total-C Total-cholesterol, LDL-C Low-density lipoprotein, HDL-C High-density lipoprotein and $\mathrm{VO}_{2 \text { peak, }}$ Peak oxygen consumption 
respectively), while there was a decrease in LDL-C over time in both groups $(p<0.001$ for time).

\section{Shift in HDL subclass distribution}

The distribution and percent change of HDL subclasses in response to the exercise intervention are presented in representative scan sections of large, intermediate and small subclass distributions (Fig. 1). At baseline, the distribution of large, intermediate and small $\mathrm{HDL}$ subclasses were not different between the control and exercise groups $(28.4 \pm 2.2 \%$ vs $26.4 \pm 1.8 \%, p=0.803$; $59.5 \pm 1.4 \%$ vs $58.5 \pm 1.0 \%, p=0.701$; and $11.8 \pm 1.5 \%$ vs $15.0 \pm 1.4 \%, p=0.112$, respectively). The distribution of large HDL subclasses did not change in response to the intervention $(p=0.105$ for interaction), while the distribution of small HDL subclasses decreased in the exercise group compared to controls $(p=0.004$ for interaction). When correcting for the change in BMI, this effect was maintained ( $p=0.040$ for interaction). The distribution of intermediate HDL subclasses was similar between groups in response to the intervention $(p=0.523$ for interaction).

\section{Changes in HDL function}

At baseline, cholesterol efflux capacity did not differ between control and exercise groups $(3.77 \pm 0.22$ vs $3.63 \pm 0.20 \mathrm{AU}, p=0.808$, respectively). HDL-mediated cholesterol efflux capacity did not change in response to a 12-week exercise intervention $(p=0.524$ for interaction, Fig. 2a). At baseline HDL anti-inflammatory function (expressed as relative reduction in VCAM expression in HUVEC cells) did not differ between control and exercise groups $(0.47 \pm 0.07$ vs $0.50 \pm 0.09 \mathrm{AU}$, $p=0.504$, respectively, Fig. $2 \mathrm{~b})$ and did not change in response to a 12-week exercise intervention $(p=0.516$ for interaction).

At baseline, serum PON activity did not differ between the control and the exercise groups $(0.90 \pm 0.07$ vs $0.83 \pm 0.05 \mathrm{U} / \mathrm{L}, p=0.173$, respectively). After 12 weeks, serum PON activity decreased in response to the exercise intervention only ( $p=0.021$ for interaction, Fig. 3a), even after adjusting for the change in BMI ( $p=0.006$ for interaction). In contrast, serum and HDL PON-1 expression did not differ at baseline between groups $(p=0.751$ and $p=0.464$, respectively,

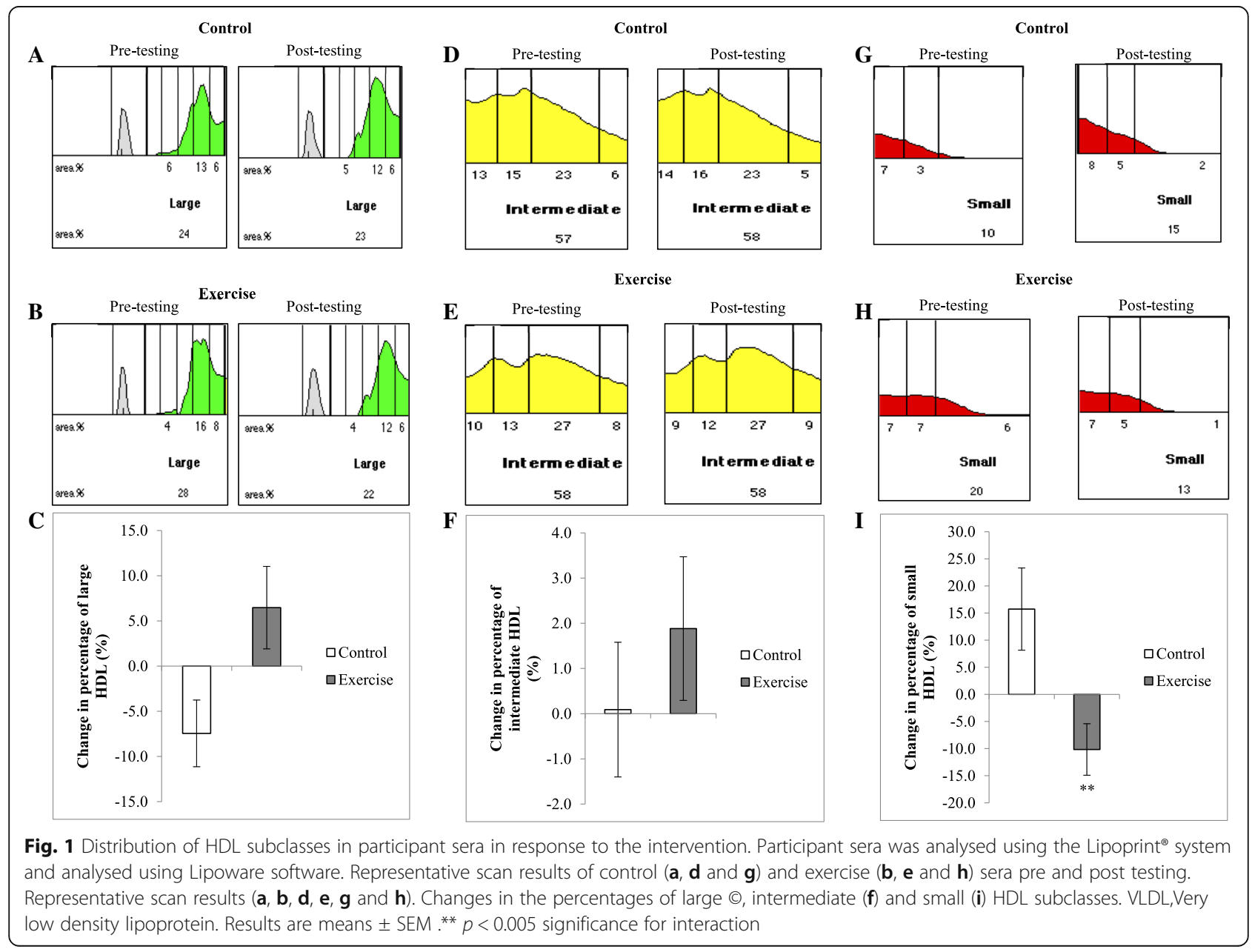




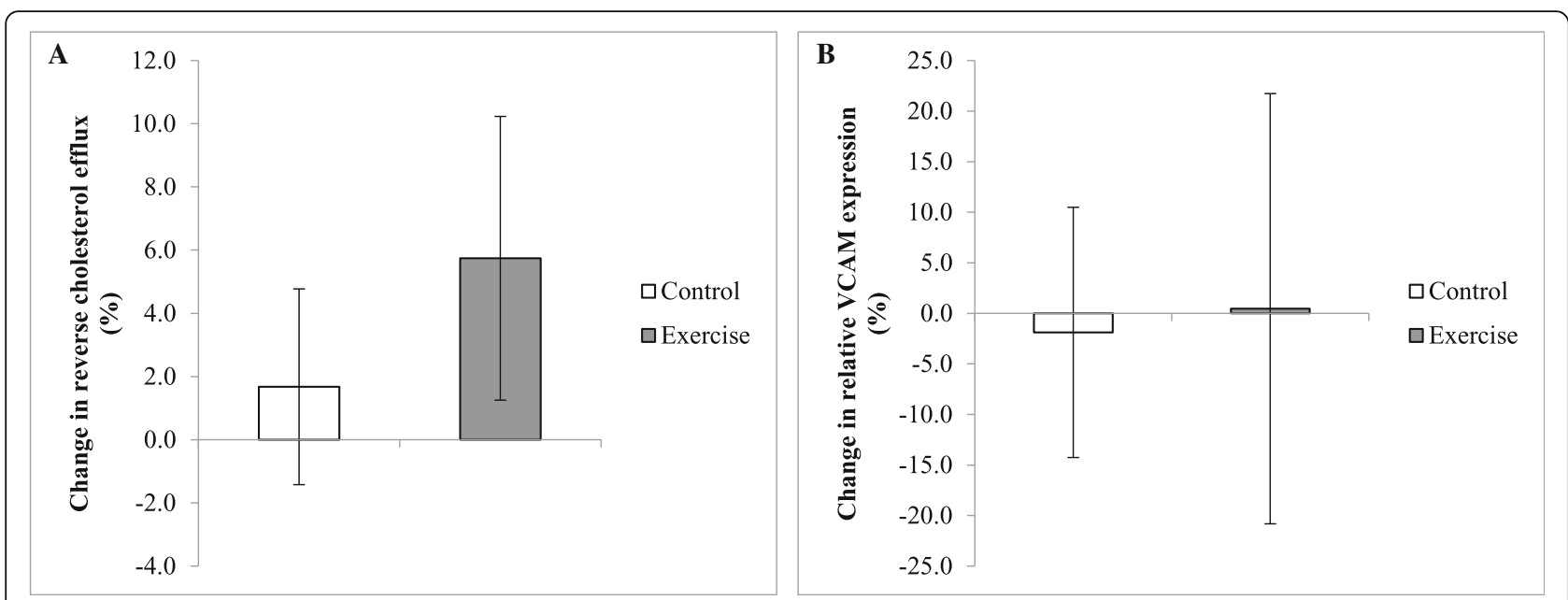

Fig. 2 Changes in reverse cholesterol efflux capacity and anti-inflammatory function in response to the intervention. [3H-Cholesterol] was effluxed from RAW264.7 cells for $4 \mathrm{~h}$ prior to scintillation counting. Cholesterol efflux capacity represents the mean radiolabel present in culture media relative to that of an untreated control (a). HUVEC cells were treated with $10 \mu \mathrm{g} / \mathrm{ml}$ participant $\mathrm{HDL}$ prior to $20 \mathrm{ng} / \mathrm{ml}$ tumour necrosis factor (TNF) treatment for 8 h. Results are calculated relative to a no-HDL treatment control (b). Cell lysates were harvested and stored in RNAprotect reagent prior to RNA extraction, followed by cDNA synthesis and quantitative real time PCR. Results as percentage changes relative to a baseline. Results are means \pm SEM. VCAM, Vascular Cell Adhesion Molecule

Fig. 3b-e) or in response to the intervention $(p=0.888$ and $p=0.697$ for interaction, respectively). The association between PON activity and expression was explored at baseline in all participants and serum PON activity were positively correlated with serum and HDL PON-1 expression $(r=0.48, p=0.016$, and $r=0.57$, $p=0.001$, respectively). However, percentage change in PON activity was not associated with change in serum and HDL PON-1 expression $(r=-0.05, p=0.817$, and $r=0.09, p=0.633$, respectively).

At baseline, serum PAF-AH activity did not differ between the control and the exercise groups $(12.7 \pm 1.4$ vs $15.2 \pm 1.2 \mathrm{U} / \mathrm{L}, p=0.311$, respectively). There was no difference in PAF-AH activity between groups in response to the intervention ( $p=0.112$ for interaction, Fig. $4 \mathrm{a})$. In contrast, serum PAF-AH expression decreased in response to the exercise intervention compared to controls $(p=0.002$ for interaction, Fig. 4d). This effect in the exercise group was maintained when correcting for the change in BMI ( $p$ $=0.003$ for interaction). However, changes in HDL PAF-AH expression were not different between groups over time ( $p=0.493$ for interaction). No associations were found between PAF-AH activity and serum and HDL PAF-AH expression at baseline $(r=0.02, p=921$, and $\mathrm{r}=$ $0.09, p=681$, respectively) or between changes in activity and expression over the 12-week intervention $(r=0.38$, $p=0.055$, and $r=0.17, p=0.441$, respectively).

\section{Relationships between anthropomorphic measures,} cardiorespiratory fitness and HDL function and subclass At baseline, higher BMI was associated with lower cholesterol efflux capacity $(r=-0.42, p=0.024)$ and less large
HDL subclasses $(r=-0.37, p=0.041$, Additional file 1: Table S1). There were no associations between the changes in aerobic capacity, body composition, and HDL-C and changes in HDL function. However, a decrease in WHR in the combined sample over the twelve-week period, was associated with an increase in the percentage of large HDL subclasses $(r=-0.39, p=0.035$ Fig. $5 b)$.

\section{Relationships between measures of HDL functionality with $\mathrm{HDL}$ subclass}

At baseline, reverse cholesterol efflux was positively associated with anti-inflammatory function $(r=0.68, p=0.016$, Additional file 1: Table S1). Similarly, higher HDL anti-inflammatory function was associated with a greater percentage of large HDL and lower percentage of intermediate HDL subclasses $(r=0.53, p=0.049$ and $r=-0.54, p=0.043$, respectively, Fig. 5a). In all participants, an increase in PON activity, over the twelve-week period, was associated with an increase in the percentage of small HDL subclasses $(r=0.51$, $p=0.004$, Fig. 5c).

\section{Discussion}

This study aimed to explore whether exercise training results in the modification of HDL functionality and HDL subclass distribution. Although HDL-C concentrations were unchanged, the 12-week exercise training intervention in obese black South African women resulted in a reduction in PON activity, serum PAF-AH expression and percentage of small HDL subclasses. For the first time, we provide evidence to suggest that exercise training may revert HDL subclass distribution back to a "non-obese" state in a black South African population. 


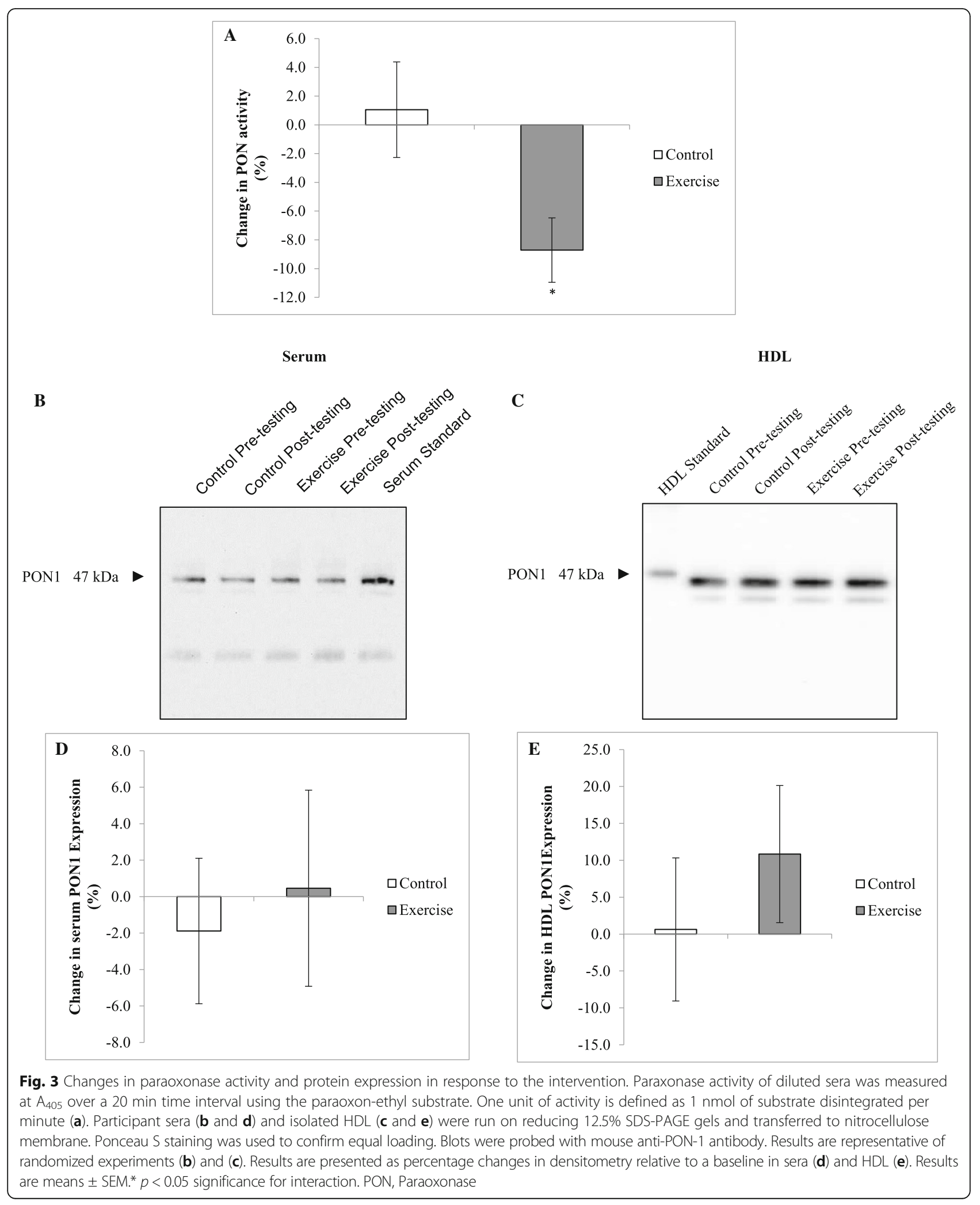




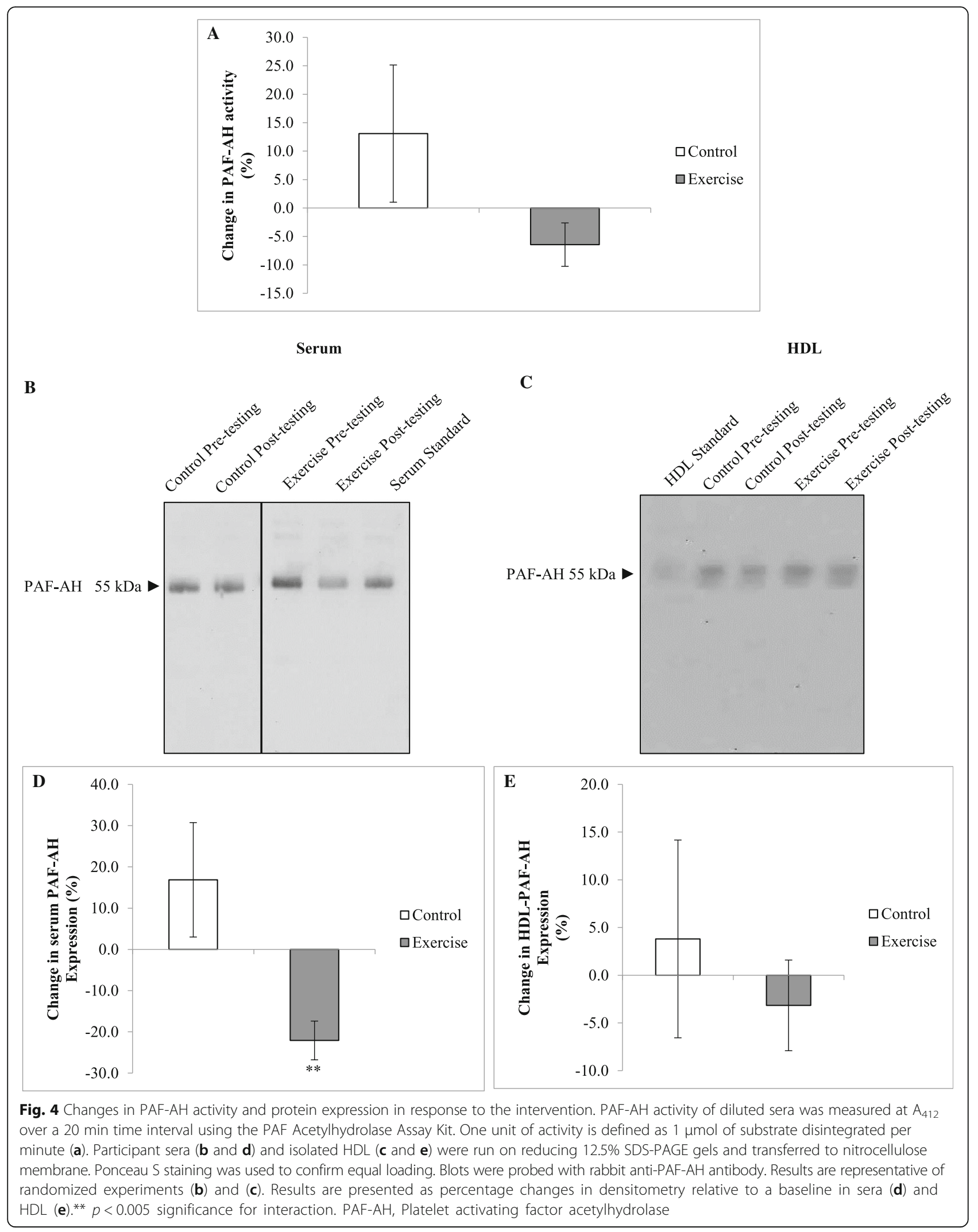



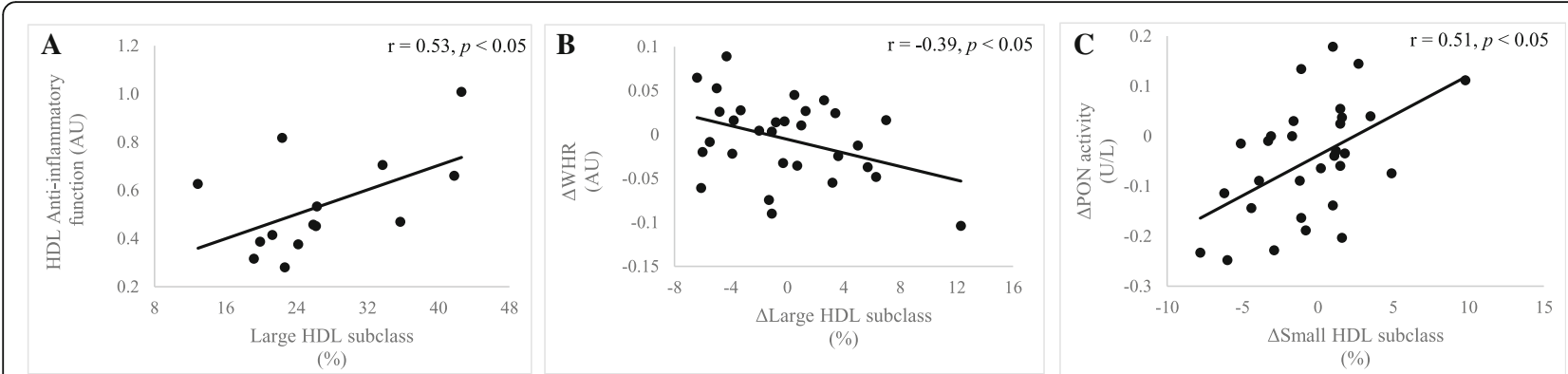

Fig. 5 Associations between HDL subclass, function and changes in body composition in response to the 12-week intervention. Baseline HDL anti-inflammatory function is plotted against baseline large HDL subclass distribution (a). Changes in WHR (b) are plotted against changes in large HDL subclass percentage. Changes in HDL function (PON activity (c)) are plotted against changes in small HDL subclass percentages. Values represent Pearson correlation coefficients. AU, Arbitrary units; WHR, Waist/hip ratio and PON, Paraoxonase

Exercise interventions are routinely prescribed for obese individuals with the aim of reducing the risk of cardiometabolic complications, and have been shown to promote an increase in HDL-C concentration [8, 31, 32]. To our knowledge, this is the first study to examine the effects of exercise training on HDL-C concentrations in a black African population, who present with lower HDL-C levels compared to other populations [19, 21]. However, the current study did not find any change in HDL-C following the intervention. Despite no changes in HDL-C concentrations, the exercise intervention resulted in a significant decrease in small HDL subclasses.

A previous study in black South African women reported that obesity was associated with lower levels of large and high levels of smaller HDL subclasses, compared to normal-weight subjects [27]. In the current study, a statistically significant change in body composition (BMI and WHR) following an exercise intervention was observed, however, these changes were minimal. Critically, when adjusting for the changes in BMI and WHR, the exercise training adaptations in HDL subclass were maintained. This suggests that changes in HDL subclass were potentially mediated by the stimulus of the exercise intervention and not necessarily through a change in body composition. These results are supported by other exercise intervention studies which have also shown that exercise training resulted in changes in HDL subclasses, favouring increases in larger HDL subclasses, that were accompanied by non-significant changes in BMI [33-36]. These results collectively suggest that exercise training may stimulate additional mechanisms which may cause shifts in HDL subclass, independent of changes in body composition.

There is a significant debate in the literature regarding the contributions of each of the HDL subclasses to overall HDL functionality. It is also important to consider that nomenclature for HDL subclass depends on the methods used for quantification and separation [37]. This study designates HDL subclasses as large, intermediate and small as quantified by the Lipoprint ${ }^{\circ}$ System. Much of the existing literature describes two principal HDL subclasses, the larger, HDL2 and smaller HDL3 [38, 39]. Whilst epidemiological studies describe lower HDL2 levels as an inverse predictor for cardiovascular disease, pre-clinical studies describe the benefits of increased HDL3 owing to a higher association with cardioprotective proteins and lipids, as reviewed by [10]. Therefore, while the results from this study are consistent with other studies in the literature, it is difficult at this stage to affirm whether the decrease in small HDL subclasses is of benefit to overall risk of CVD. This requires further investigation.

Common mechanisms stimulated by exercise training, including reductions in oxidative stress and inflammation [40, 41], may be contributing factors to the shift in HDL subclass, observed in the current study. The exercise intervention resulted in a decrease in the antioxidative activity of PON and the serum expression of PAF-AH, respectively. This is in contrast to other studies that found improvements in PON activity and overall HDL antioxidant function after moderate aerobic exercise interventions (3 or 4 months) in metabolic syndrome and type 2 diabetic patients, respectively $[35,36]$. The unexpected PON activity results in the present study may be explained by the participants being normolipidemic and nondiabetic prior to the intervention, and/or differences in the type and intensity of the exercise intervention. PON activity is largely modulated by genetic and environmental factors such as smoking and intake of antioxidants, and these factors may also contribute to differences between studies [35, 36, 42]). Indeed, studies in overweight adolescents and type 2 diabetic patients have reported that exercise training interventions may reduce oxidative stress and the potential risk of CVD [43]. This suggests that the beneficial aspects of exercise training may result in a compensatory reduction in PON activity owing to reduction in oxidative stress.

HDL not only controls oxidative stress but also performs several anti-inflammatory functions [17]. Here, 
the exercise intervention did not improve HDL anti-inflammatory function. In contrast, only one other study has explored how a 21-day dietary and exercise intervention in obese men (which resulted in a $3.2 \%$ decrease in BMI), improved HDL anti-inflammatory function [35]. Despite a low sample size, our data do not support an association between changes in BMI or WHR with a change in anti-inflammatory function. In addition, the minimal changes in body composition observed in the current study may indeed suggest that substantial changes in body composition may be required to influence changes in HDL anti-inflammatory function.

The current study showed that exercise training stimulated a decrease in the serum expression of PAF-AH. Previous literature has reported increased PAF-AH activity in response to a short term (3 weeks) diet and exercise intervention in obese participants [44]. This suggests that diet and exercise-based interventions stimulate different mechanisms of PAF-AH activity, which may be specific to weight loss, changes in body composition and/or change in diet quality. In the current study, there were no associations between changes in PAF-AH activity and expression with changes in BMI and WHR. Furthermore, participants did not display a substantial change in weight, thus the decrease in PAF-AH expression may relate to other mechanisms specifically associated with an exercise training stimulus; however, further research is required in this area. The lack of a significant correlation between HDL and serum PAF-AH expression may further explain this disparity.

Cholesterol efflux capacity is considered to be the primary function of HDL in vivo and this did not change following the 12-week intervention. These results are consistent with previous studies conducted in African American populations where a 6 month diet programme of reduced fat and energy, combined with low-intensity exercise, showed improvements in fitness and weight loss, but no changes in cholesterol efflux capacity [45]. Baseline results of the current study showed that cholesterol efflux capacity was associated with a lower WHR. Similarly, a study, examining the relationship between body composition and HDL cholesterol efflux, indicated that an increase in waist circumference was an accurate predictor of impairment in cholesterol efflux capacity [46]. Previously, an association between increased BMI and lower cholesterol efflux capacity has been shown in obese subjects [47]. Commensurate with these findings, improvement in cholesterol efflux capacity in individuals undergoing exercise interventions were only significant in those individuals with significant weight loss [48]. Accordingly, the lack of clinically significant changes in body composition in our study may explain the lack of change in HDL cholesterol efflux capacity in response to the intervention.

The present study highlights that HDL function and subclass may be modified concurrently in response to exercise training. Accordingly, it is recommended that both HDL function and subclass are considered when assessing changes in CVD risk in response to an exercise training intervention. We report an association between a decrease in small HDL subclasses and a decrease in PON activity. PON is preferentially associated with smaller HDL subclasses, therefore suggesting that a decrease in PON activity is associated with a decrease in the distribution of small HDL subclasses [49]. HDL subclasses have inherent functional differences; however, few studies have considered the associations between HDL size and function and have not done so using the Lipoprint ${ }^{\circ}$ System. Therefore, this study presents novel findings that changes in traditional measures of HDL function can be linked to new measures of HDL size.

Notably, this study has limitations that must be considered. In particular, this study was limited with a relatively small sample size. Whilst cholesterol efflux measurements were performed using biological triplicate measurements for each participant, due to technical constraints, the anti-inflammatory assay was only able to be performed on 8 participants. Furthermore, other clinical studies normally employ J744 macrophage cells to test cholesterol efflux capacity. RAW264.7 cells were optimized for the conditions employed in this study and produced reproducible results and therefore presented an applicable model. There was, however, good adherence to the exercise training, thus allowing for adequate interpretation of its effects on HDL function and subclass distribution.

\section{Conclusion}

Despite no change in HDL-C concentrations, our study presents novel findings that exercise training may revert the HDL subclass distribution to a "non-obese" state in obese black women. Furthermore, exercise training altered HDL antioxidative and anti-thrombotic function, independent of changes in body composition. This study provides novel evidence on the association between HDL function and subclass distribution in a black African population, suggesting that studying HDL subclass and function may be a sensitive approach to assess CVD risk in this population compared to the measurement of HDL-C levels alone.

\section{Additional file}

\footnotetext{
Additional file 1: Figure S1. Breakdown of HDL analysis in control and exercise groups. The distribution of participants into exercise and control groups is presented. Further divisions for assessment of HDL function and subclass are expanded. Table S1. Associations between HDL functionality and subclass measures with body composition and HDL-C in all participants at baseline. Values are Pearson correlation coefficients. BMI, Body mass index; WHR, Waist/hip ratio; $\mathrm{VO}_{2 \text { peak, }}$ Peak oxygen consumption; HDL-C, High-density lipoprotein; PON, Paraoxonase and PAF-AH, Platelet activating factor acetylhydrolase ${ }^{*} p<0.05,{ }^{* *} p<0.005$. (DOCX $27 \mathrm{~kb}$ )
} 


\section{Abbreviations}

ACAT: Acyl-CoA cholesterol acyltransferase; AU: Arbitary units; BMl: Body mass index; CPM: Counts per minute; CVD : Cardiovascular disease; HDL: High-density lipoprotein; HDL-C: High-density lipoprotein cholesterol; $H R_{\max }$ : Maximal heart rate; HUVEC : Human umbilical vein endothelial cells; LDL: Low-density lipoprotein; LDL-C: Low-density lipoprotein cholesterol; MEM: Minimum essential eagle; PAF-AH : Platelet activating factor acetylhydrolase; PON : Paraoxonase; SDS-PAGE: Sodium dodecyl sulfate polyacrylamide gel electrophoresis; SEM: Standard error of mean; TNFa: Tumour necrosis factor alpha; VCAM : Vascular cell adhesion molecule; VEGF: Vascular endothelial growth factor; VLDL: Very low-density lipoprotein; $\mathrm{VO}_{2 \text { max }}$ : Maximal oxygen consumption; WHR: Waist/hip ratio

\section{Acknowledgements}

We thank Hendriena Victor, Keitumetse (Tumi) Smouse, Louise Clamp, Lindokuhle Phiri, Nandipha Sinyanya and Ntombekhaya Zoneleni from the Department of Human Biology at the University of Cape Town for their assistance in sample collection and recruitment. We thank Roshan Ebrahim and Kate Larmuth from the Division of Medical Biochemistry, Institute of Infectious Disease and Molecular Medicine at the University of Cape Town for their technical assistance with scintillation counting.

\section{Funding}

This study was funded by the National Research Foundation (Grant numbe 93577), the South African Medical Research Council and the Swiss South African Joint Research Programme (JRP16) to SL and RWJ and the University of Cape Town.

\section{Availability of data and materials}

The datasets used and/or analysed during the current study are available from the corresponding author on reasonable request.

\section{Authors' contributions}

AEM and JHD conceptualized the overall intervention design and coordinated sample collection. NJW assisted in sample collection, performed all analytical tests and analysis and compiled and edited the manuscript. SL provided the lab space and equipment for experiments. AK provided lab space and training for radioactive experiments. All authors read and approved the manuscript.

\section{Ethics approval and consent to participate}

This study was approved by the Human Research Ethics Committee at the University of Cape Town (HREC REF: 054/2015), complies with Declaration of Helsinki principles and participants provided written consent prior to testing. Clinical trials number: PACTR201711002789113.

\section{Consent for publication}

Strict confidentiality of study participants was maintained throughout and no individual data is presented in the study.

\section{Competing interests}

The authors declare that they have no competing interests.

\section{Publisher's Note}

Springer Nature remains neutral with regard to jurisdictional claims in published maps and institutional affiliations.

\footnotetext{
Author details

${ }^{1}$ Hatter Institute for Cardiovascular Research in Africa, Department of Medicine, Faculty of Health Sciences, University of Cape Town, Cape Town, South Africa. ${ }^{2}$ Non-Communicable Diseases Research Unit, South African Medical Research Council, Cape Town, South Africa. ${ }^{3}$ UCT Research Unit for Receptor biology, Department of Integrative Biomedical Sciences and Institute of Infectious Disease and Molecular Medicine, Faculty of Health Sciences, University of Cape Town, Cape Town, South Africa. ${ }^{4}$ Division of Exercise Science and Sports Medicine, Department of Human Biology, University of Cape Town, Cape Town, South Africa.
}

Received: 24 August 2018 Accepted: 27 September 2018

Published online: 10 October 2018

\section{References}

1. World Health Organization. World Health Organization: Fact Sheet: Obesity and Overweight [Internet]. 2014. Available from: www.who.int/mediacentre/ factsheets/fs311/en/. Accessed 2017

2. Curioni CC, Lourenco PM. Long-term weight loss after diet and exercise: a systematic review. Int J Obes. 2005;29:1168-74.

3. Hawley JA. Exercise as a therapeutic intervention for the prevention and treatment of insulin resistance. Diabetes Metab Res Rev. 2004;20:383-93.

4. Lavie CJ, Milani RV. Effects of cardiac rehabilitation, exercise training, and weight reduction on exercise capacity, coronary risk factors, behavioral characteristics, and quality of life in obese coronary patients. Am J Cardiol. 1997;79:397-401.

5. Ohta M, Nanri H, Matsushima Y, Sato Y, Ikeda M. Blood pressure-lowering effects of lifestyle modification: possible involvement of nitric oxide bioavailability. Hypertens Res. 2005;28:779-86.

6. Wood PD, Stefanick ML, Dreon DM, et al. Changes in plasma lipids and lipoproteins in overweight men during weight loss through dieting as compared with exercise. N Engl J Med. 1988;319:1173-9.

7. Slentz CA, Duscha BD, Johnson JL, et al. Effects of the amount of exercise on body weight, body composition and measures of central obesity. Arch Intern Med Med. 2004:164:31-9.

8. Argani N, Sharifi G, Golshahi J. Comparison of the effect of different intensity exercise on a bicycle ergometer on postprandial lipidemia in type II diabetic patients. Abstract Original Article. 2014;10:147-53.

9. Gordon T, Castelli WP, Hjortland MC, Kannel WB, Dawber TR. High density lipoprotein as a protective factor against coronary heart disease: the Framingham study. Am J Med. 1977;62:707-14.

10. Woudberg NJ, Pedretti S, Lecour S, et al. Pharmacological intervention to modulate HDL: what do we target? Front Pharmacol. 2018;8:1-16.

11. Woudberg NJ, Goedecke JH, Lecour S. Protection from Cardiovascular Disease Due to Increased High-Density Lipoprotein Cholesterol in African Black Populations: Myth or Reality. Ethn Dis. 2016:26:553-60.

12. Kühnast S, Fiocco M, van der Hoorn JWA, Princen HMG, Jukema JW. Innovative pharmaceutical interventions in cardiovascular disease: focusing on the contribution of non-HDL-C/LDL-C-lowering versus HDL-C-raisingA systematic review and meta-analysis of relevant preclinical studies and clinical trials. Eur J Pharmacol. 2015;763:48-63.

13. Luscher TF, von Eckardstein A, Simic B. Therapeutic targets to raise HDL in patients at risk or with coronary artery disease. Curr Vasc Pharmacol. 2012; 10:720-4.

14. Chang Tl, Streja E, Moradi H. Could high-density lipoprotein cholesterol predict increased cardiovascular risk? Curr Opin Endocrinol Diabetes Obes. 2017:24:140-7.

15. Rader DJ, Tall AR. The not-so-simple HDL story: Is it time to revise the HDL cholesterol hypothesis? Nat Med. 2012;18:1344-6.

16. Barter PJ, Rye KA. High density lipoproteins and coronary heart disease. Atherosclerosis. 1996:121:1-12.

17. Nofer J-R, Kehrel B, Fobker M, et al. HDL and arteriosclerosis: beyond reverse cholesterol transport. Atherosclerosis. 2002;161:1-16.

18. Camont L, Lhomme M, Rached F, et al. Small, dense high-density Lipoprotein-3 particles are enriched in negatively charged phospholipids relevance to cellular cholesterol efflux, Antioxidative, antithrombotic, antiinflammatory, and Antiapoptotic functionalities. Arterioscler Thromb Vasc Biol. 2013;33:2715-23.

19. Sliwa K, Wilkinson D, Hansen C, et al. Spectrum of heart disease and risk factors in a black urban population in South Africa (the heart of Soweto study): a cohort study. Lancet. 2008;371:915-22.

20. Ellman N, Keswell D, Collins M, Tootla M, Goedecke JH. Ethnic differences in the association between lipid metabolism genes and lipid levels in black and white South African women. Atherosclerosis Elsevier Ltd. 2015;240:311-7.

21. Goedecke JH, Utzschneider K, Faulenbach MV, et al. Ethnic differences in serum lipoproteins and their determinants in South African women. Metabolism. 2010:59:1341-50.

22. Punyadeera $\mathrm{C}$, van der Merwe MT, Crowther NJ, et al. Ethnic differences in lipid metabolism in two groups of obese South African women. J Lipid Res. 2001;42:760-7. 
23. Blazek A, Rutsky J, Osei K, Maiseyeu A, Rajagopalan S. Exercise-mediated changes in high-density lipoprotein: Impact on form and function. Am Heart J Mosby Inc. 2013;166:392-400.

24. Goedecke JH, Mendham AE, Clamp L, et al. An exercise intervention to unravel the mechanisms underlying insulin resistance in a cohort of black South African women: Protocol for a randomized controlled trial. JMIR Res Protoc. 2018;7(4):e75.

25. Norton K, Olds T, Norton Kl. Anthropometrica: a textbook of body measurement for sports and health courses. Sydney: UNSW Press; 1996.

26. Brulhart-Meynet MC, Braunersreuther V, Brinck J, et al. Improving reconstituted $\mathrm{HDL}$ composition for efficient post-ischemic reduction of ischemia reperfusion injury. PLoS One. 2015;10:1-16.

27. Woudberg NJ, Goedecke JH, Blackhurst D, et al. Association between ethnicity and obesity with high-density lipoprotein (HDL) function and subclass distribution. Lipids Health Dis. 2016;15:92.

28. Markwell MAK, Haas SM, Bieber LL, Tolbert N. A modification of the Lowry procedure to simplify protein determination in membrane and lipoprotein samples. Anal Biochem. 1978;87:206-10.

29. Sankaranarayanan S, Kellner-Weibel G, de la Llera-Moya M, et al. A sensitive assay for ABCA1-mediated cholesterol efflux using BODIPY-cholesterol. J Lipid Res. 2011;52:2332-40.

30. Ortega JF, Hamouti N, Fernández-Elías VE, Mora-Rodriguez R. Comparison of glucose tolerance tests to detect the insulin sensitizing effects of a bout of continuous exercise. Appl Physiol Nutr Metab. 2014;39:787-92.

31. Kelley GA, Kelley KS. Aerobic exercise and lipids and lipoproteins in men: a metaanalysis of randomized controlled trials. J Mens Health Gend. 2006;3:61-70.

32. Kodama S, Tanaka S, Saito K, et al. Effect of aerobic exercise training on serum levels of high-density lipoprotein cholesterol. Arch Intern Med. 2007; 167:999-1008

33. Sarzynski MA, Burton J, Rankinen T, et al. The effects of exercise on the lipoprotein subclass profile: a meta-analysis of 10 interventions. Atherosclerosis. 2015;243:364-72.

34. Riedl I, Yoshioka M, Nishida Y, et al. Regulation of skeletal muscle transcriptome in elderly men after 6weeks of endurance training at lactate threshold intensity. Exp Gerontol. 2010;45:896-903.

35. Casella-Filho A, Chagas ACP, Maranho RC, et al. Effect of exercise training on plasma levels and functional properties of high-density lipoprotein cholesterol in the metabolic syndrome. Am J Cardiol Elsevier Inc. 2011;107:1168-72.

36. Ribeiro ICD, Iborra RT, Neves MQTS, et al. HDL Atheroprotection by aerobic exercise training in type 2 diabetes mellitus. Med Sci Sports Exerc. 2008;40:779-86.

37. Asztalos BF, Tani M, Schaefer EJ. Metabolic and functional relevance of HDL subspecies. Curr Opin Lipidol. 2011;22:176-85.

38. Rosenson RS, Brewer HB, Chapman MJ, et al. HDL measures, particle heterogeneity, proposed nomenclature, and relation to atherosclerotic cardiovascular events. Clin Chem. 2011;57:392-410.

39. Williams PT, Krauss RM, Vranizan KM, et al. Associations of lipoproteins and apolipoproteins with gradient gel electrophoresis estimates of high density lipoprotein subfractions in men and women. Arterioscler Thromb Vasc Biol. 1992;12:332-40.

40. Siti HN, Kamisah Y, Kamsiah J. The role of oxidative stress, antioxidants and vascular inflammation in cardiovascular disease (a review). Vascul Pharmacol Elsevier Inc. 2015;71:40-56.

41. Vinetti $G$, Mozzini $C$, Desenzani $P$, et al. Supervised exercise training reduces oxidative stress and cardiometabolic risk in adults with type 2 diabetes: a randomized controlled trial. Sci Rep. 2015;5:9238.

42. da Costa Vieira JL, Gomes ME, Almeida AB, Moriguchi EH. Changes in the profile of lipoprotein subfractions associated with hormone replacement therapy. Arq Bras Cardiol. 2001;76:177-88.

43. Li C, Feng F, Xiong $X$, Li R, Chen N. Exercise coupled with dietary restriction reduces oxidative stress in male adolescents with obesity. J Sports Sci. 2017;35:663-8.

44. Roberts CK, Ng C, Hama S, Eliseo AJ, Barnard RJ. Effect of a short-term diet and exercise intervention on inflammatory/anti-inflammatory properties of HDL in overweight/obese men with cardiovascular risk factors. J Appl Physiol. 2006;101:1727-32.

45. Aicher BO, Haser EK, Freeman LA, et al. Diet-Induced Weight Loss in Overweight or Obese Women and Changes in High-Density Lipoprotein Levels and Function. Obesity. 2012;20:2057-62.

46. Attia N, Fournier N, Vedie B, et al. Impact of android overweight or obesity and insulin resistance on basal and postprandial SR-BI and ABCA1-mediated serum cholesterol efflux capacities. Atherosclerosis. 2010;209:422-9.
47. Sasahara T, Nestel P, Fidge N, Sviridov D. Cholesterol transport between cells and high density lipoprotein subfractions from obese and lean subjects. J Lipid Res. 1998;39:554.

48. Lesna IK, Suchanek P, Kovar J, Poledne R. Life style change and reverse cholesterol transport in obese women. Physiol Res. 2009;58:S33-8.

49. Sean Davidson W, Silva RAGD, Chantepie S, et al. Proteomic analysis of defined HDL subpopulations reveals particle-specific protein clusters: relevance to antioxidative function. Arterioscler Thromb Vasc Biol. 2009;29: 870-6.

Ready to submit your research? Choose BMC and benefit from:

- fast, convenient online submission

- thorough peer review by experienced researchers in your field

- rapid publication on acceptance

- support for research data, including large and complex data types

- gold Open Access which fosters wider collaboration and increased citations

- maximum visibility for your research: over $100 \mathrm{M}$ website views per year

At $\mathrm{BMC}$, research is always in progress.

Learn more biomedcentral.com/submissions 